
\section{Damage on Metallic Surfaces Analyzed by Unidirectional Laser Oblique Illumination (ULOI)}

Temporal Evolution of Incipient

\author{
Eduardo A. Favret ${ }^{1,2}$, Néstor O. Fuentes ${ }^{2,3}$ \\ and Lisandro Ferrero ${ }^{2}$
}

${ }^{1}$ Centro de Investigaciones de Recursos Naturales (CIRN). Instituto de Tecnología Agropecuaria (INTA),. Buenos Aires. Argentina. eafavret@cnia.inta.gov.ar

${ }^{2}$ Univ. Nacional de Gral San Martin, Buenos Aires. Argentina. ${ }^{3}$ Comisión Nacional de Energía Atómica, Buenos Aires. Argentina. Nestor.Fuentes@cnea.gov.ar

\section{Introduction}

To one degree or another, most materials experience some type of interaction with a large number of diverse environments. Often, such interactions impair a material's usefulness as a result of the deterioration of its mechanical properties (e.g., ductility and strength), other physical properties, or appearance. Deteriorative mechanisms are different for metals, ceramic materials, and polymers. In metals, there is actual material loss either by dissolution (corrosion) or by the formation of non-metallic scale or film (oxidation).

Everyday objects and structures are made of well known metals like aluminium, brass, bronzes, copper, titanium, zinc. As an example, some of their applications can be mentioned:

- Aluminium: aircraft structural parts, food / chemical handling and storage equipment, heat exchangers, pressure vessels and piping, beverage cans, bus bodies, automotive parts (engine blocks, pistons and manifolds).

- Brass: automotive radiator cores, ammunition components, lamp fixtures, flashlight shells, kickplates.

- Bronzes: jet aircraft landing gear bearings and bushings, springs, and surgical and dental instruments.

- Copper (Electrolytic tough pitch): electrical wire, rivets, gaskets, pans, nail, and roofing.

- Titanium: airplane structures, space vehicles, high-strength prosthetic implants, petroleum and chemical industries.

- Zinc: padlocks, automotive parts (door handles and grilles), office equipment.

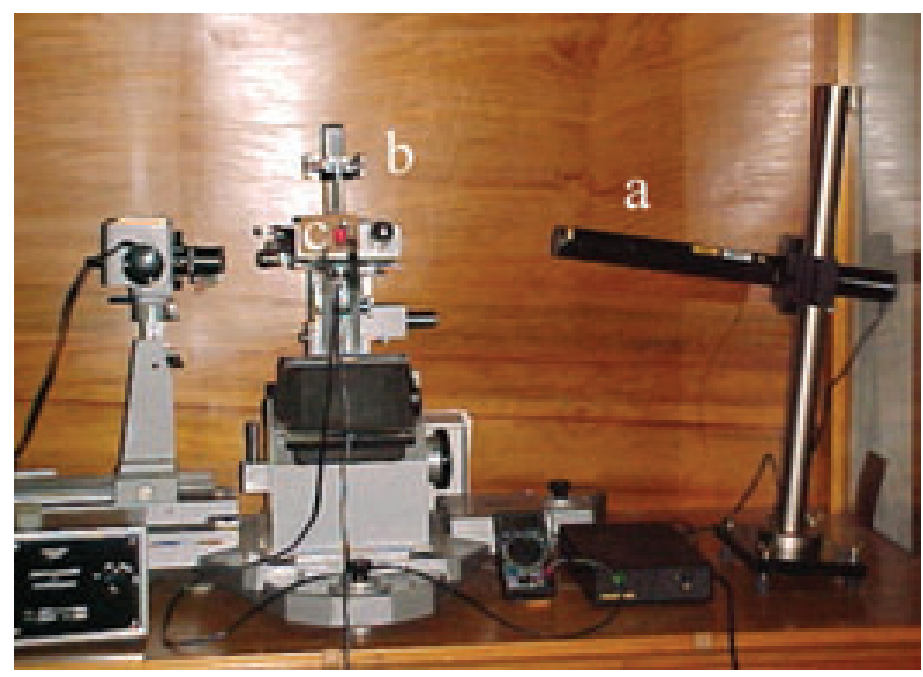

Figure 1: ULOI experimental device. a: He-Ne laser, b: sample and c: phototransistor.

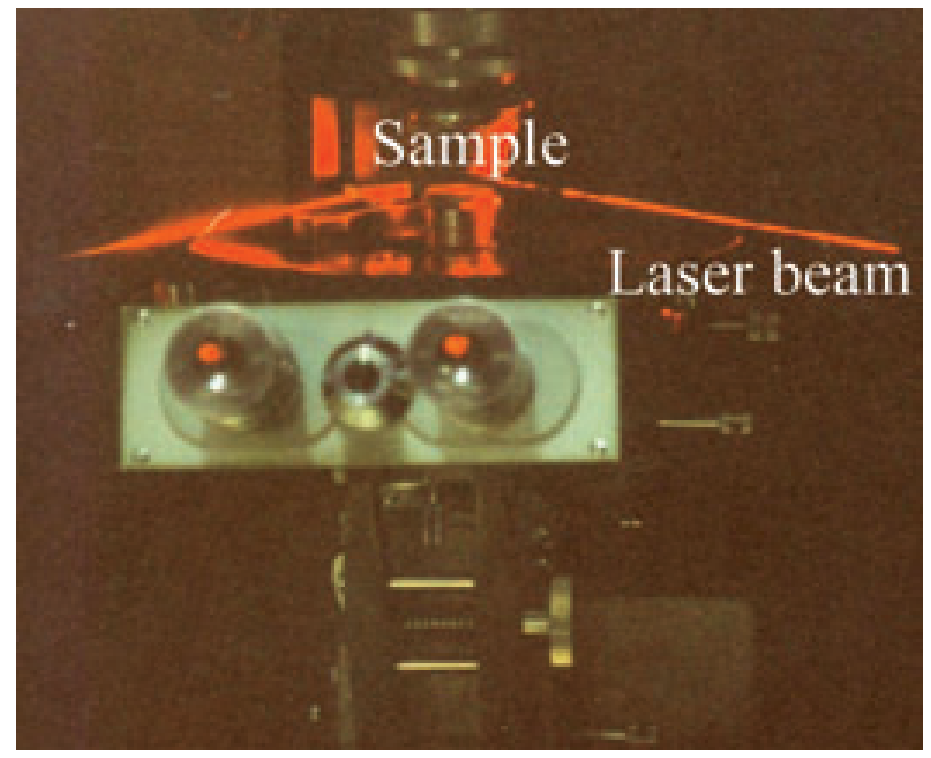

Figure 2: A close-up of part of Figure 1, taken in a darkened room showing the laser light path.

In all of these cases, corrosion due to environmental agents shows characteristic topographic patterns with distinctive orientation according to the different origin and evolution times of damage produced on their surfaces.

Corrosion is defined as the destructive and unintentional attack of a metal; it is electrochemical and ordinarily begins at the surface. The problem of metallic corrosion is one of significant proportions; in economic terms, it has been estimated that approximately $5 \%$ of an industrialized nation's income is spent on corrosion prevention and the maintenance or replacement of products lost or contaminated because of corrosion reactions.

Corrosive environments include the atmosphere, aqueous solutions, soils acids, bases, inorganic solvents, molten salts, liquid metals and, last but not least, the human body. Moisture containing dissolved oxygen is the primary corrosive agent, but other substances, including sulphur compounds (acid rain) and sodium chloride (marine atmosphere), may also contribute. Water environments can also have a variety of compositions and corrosion characteristics. Seawater contains approximately $3.5 \%$ salt (predominantly sodium chloride), as well as some minerals and organic matter. Seawater is generally more corrosive than fresh water, frequently producing pitting and crevice corrosion. Cast iron, steel, aluminium, copper and brass are generally suitable for fresh water use, whereas titanium, brass, some bronzes, copper-nickel alloys, and nickel-chromium-molybdenum alloys are highly corrosion resistant in seawater. Soils have a wide range of compositions and susceptibilities to corrosion. Compositional variables include

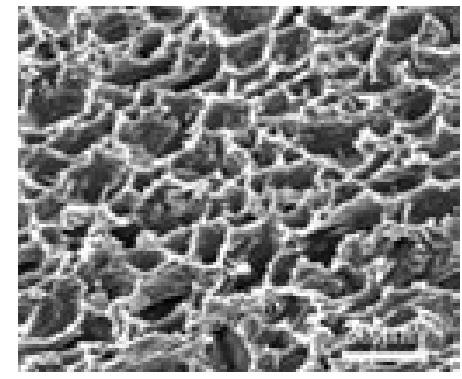

Figure 3: Zinc sample after chemical etching (150 s) with $\mathrm{HCl}$ 15\%. Crystalline grain A. SEM

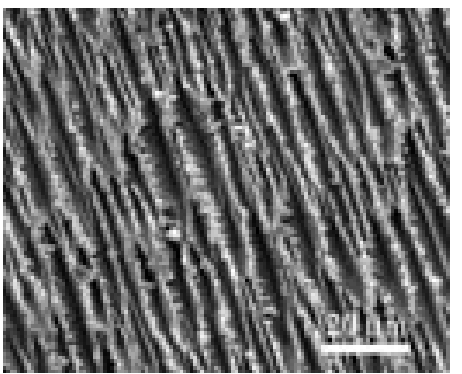

Figure 4: Zinc sample after chemical etching (150 s) with $\mathrm{HCl}$ $15 \%$. Crystalline grain B. SEM 


\section{CUSTOMIZING}

TO YOUR SPECIFIC NEEDS

Micro-manipulators, proparation materials, darkroom and general lab supplies, books, grids and apertures. Many items are manufactured in our machine shop, so customizing to your specific need is not a problem.

Some of the accessories and laboratory supplies we can supply are tweezers. tools. TEM CCD imaging systems. tensile testers, turbo evaporators. sputter coaters, substages, specimen holders, standards, carbon coaters, and more.
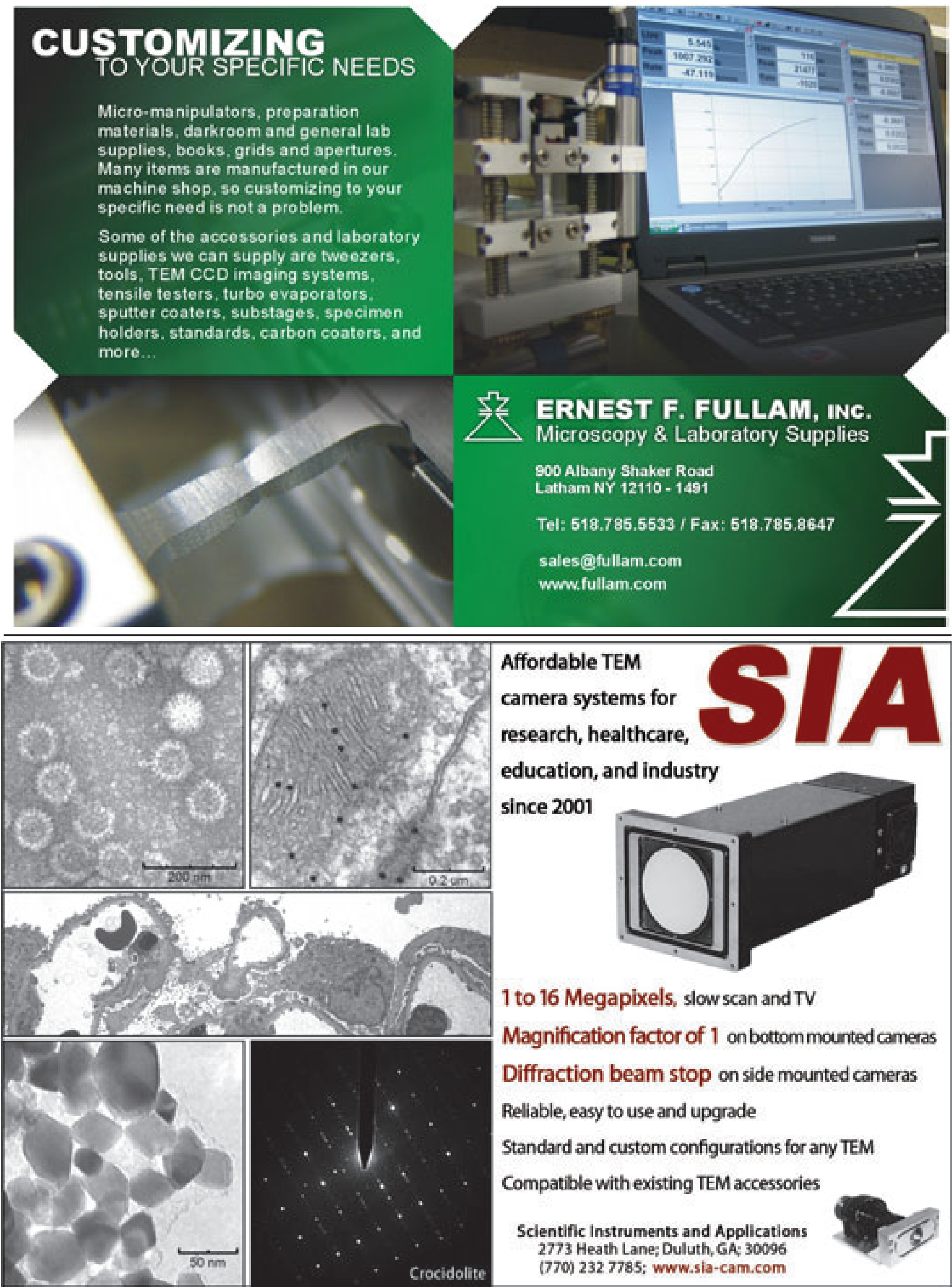

\section{Affordable TEM} camera systems for research, healthcare,

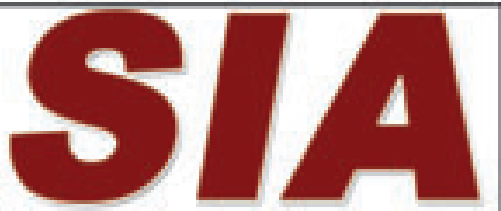
education, and industry since 2001

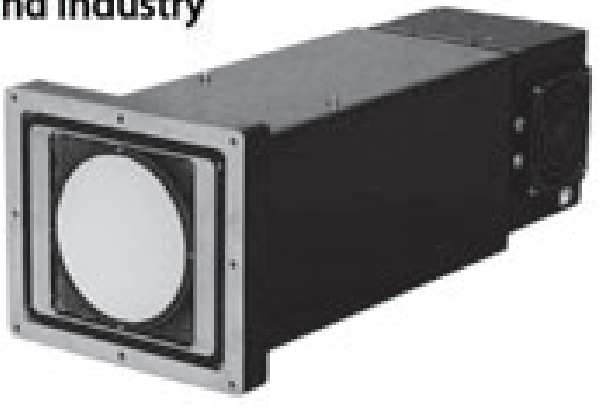

1 to 16 Megapixels, slow scan and TV

Magnification factor of 1 on bottommounted cameras Diffraction beam stop on side mounted cameras Reliable, easy to use and upgrade Standard and custom configurations for any TEM Compatible with existing TEM accessories

Scientific Instruments and Applications 2773 Heath Lane; Duluth, GA; 30096 (770) 232 7785; www.sia-cam.com

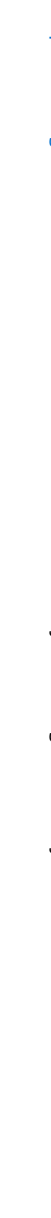

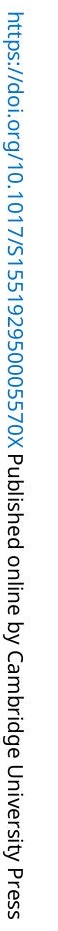

.




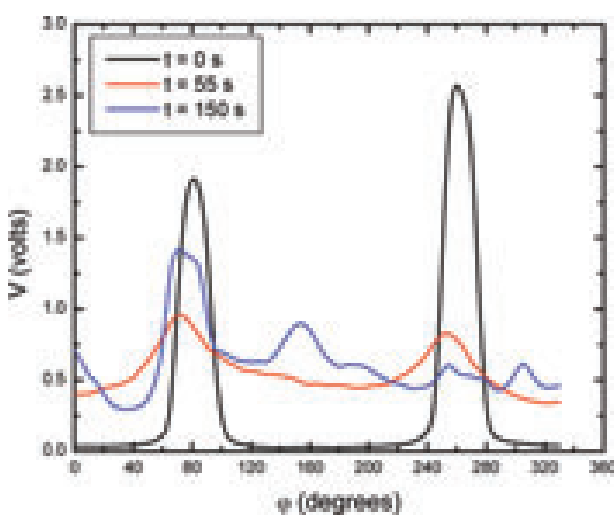

Figure 5: ULOI curve of grain A. $t$ : etching time.

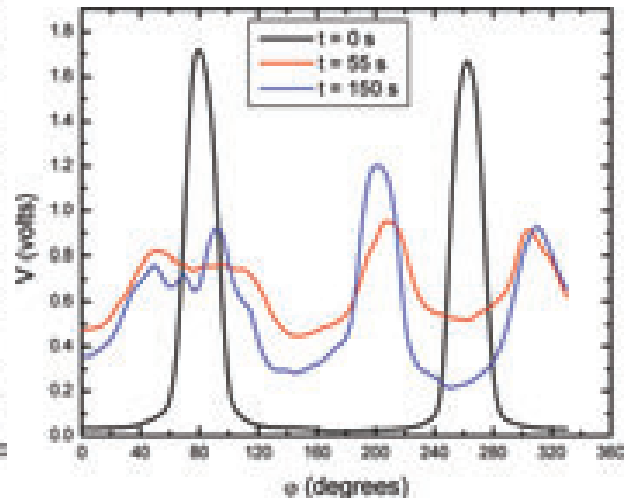

Figure 6: ULOI curve of grain B. $t$ : etching time.

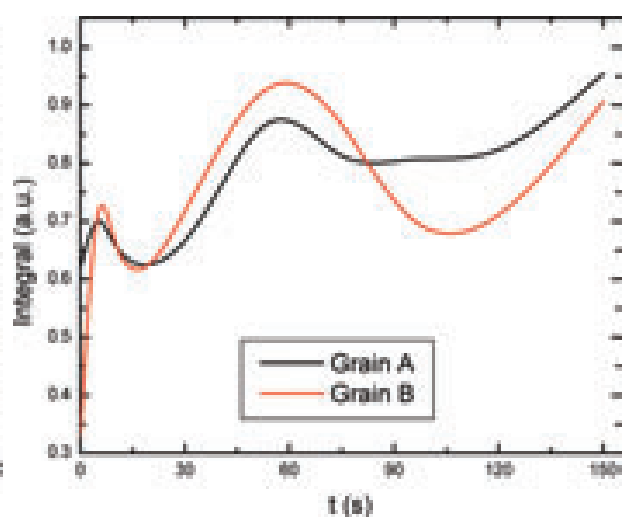

Figure 7: TEC curves of grains $A$ and $B$. moisture, oxygen, salt content, alkalinity, and acidity, as well as the presence of various forms of bacteria.

Knowledge of the nature and evolution of patterns gives a valuable insight into the atomic processes involved in the action of environmental agents, and are precisely the development and control of these processes that have a great industrial significance. Furthermore, the early detection of damage in all those surfaces exposed to environmental agents is of fundamental importance to control and evaluate corrosion performance of infrastructure materials.

In the present paper, we introduce and discuss a new application of Unidirectional Laser Oblique Illumination (ULOI). We analyze the temporal evolution of damage in surfaces by means of mathematically integrating the intensity $I(\varphi)$ with respect to the angular variable $\varphi$. Crystalline grains of zinc and aluminium were studied for different chemical reagents and the $I(\varphi)$ curves were recorded for each time of etching. Then the integral data of the ULOI curve, which we call the temporal evolution curve (TEC), was plotted versus etching time.

\section{ULOI}

During the past 30 years, the use of laser light has produced remarkable advances in microscopy. In our case, laser light has been used as a source of illumination in the metallographic microscope. The illumination system of the microscope was replaced by a He-Ne laser beam, using an angle of incidence between $5^{\circ}$ and $15^{\circ}$ with respect to the surface of the sample (Figures 1 and 2). We analyzed the variation of the intensity $(I)$, measured in volts with a phototransistor of the laser light dispersed by the surface of a crystalline grain as a function of the angle of rotation $(\varphi)$ of the sample around a perpendicular axis to its surface. This fact is partially related to the crystalline orientation of the grain as well as with the chemical solution used to reveal the structure of the material. We named this technique of illumination Unidirectional Laser Oblique Illumination (ULOI) (Favret et al., 1999; Favret and Povolo, 2001; Favret, 2002). Each maximum of the $I(\varphi)$ curve corresponds to a principal direction of surface topography. The main factor of this illumination is not necessarily the precise observation of the surface roughness, which is partially harmed by the speckle produced by the high coherence of the laser, but the possibility of detecting some surface structure that is below the resolving power of the objective.

\section{Materials}

All the samples were mechanically ground with emery paper down to 1,500 grit (granulometry of $2 \mu \mathrm{m}$ ). The surface of the samples were then subjected to different chemical solutions. Polycrystalline zinc was etched using a reagent composed by $\mathrm{HCl} 15 \%$, $\mathrm{H}_{2} \mathrm{O} 85 \%\left(7.5 \mathrm{ml} \mathrm{HCl}\right.$ and $\left.42.5 \mathrm{ml} \mathrm{H}_{2} \mathrm{O}\right)$. Polycrystalline aluminium was etched using two solutions. One was the Keller reagent, a chemical solution commonly used metallographically to reveal crystalline grains (see Table I); the other solution was $\mathrm{NaOH} 10 \%$, $\mathrm{H}_{2} \mathrm{O} 90 \%$ (caustic).

\begin{tabular}{|c|c|c|c|c|c|}
\hline \multicolumn{5}{|c|}{ Table I } \\
\hline Components & $\mathbf{H}_{2} \mathbf{O}$ & $\mathrm{HNO}_{3}$ & $\mathbf{H C l}$ & $\mathrm{HF}$ & Total \\
\hline Keller & $25 \mathrm{ml}$ & $12.5 \mathrm{ml}$ & $7.5 \mathrm{ml}$ & $2.5 \mathrm{ml}$ & $47.5 \mathrm{ml}$ \\
\hline
\end{tabular}

\section{Results}

\section{Zinc}

ULOI curves were obtained for etching times between 0 and 150 s. Crystalline grains were already observed after 10 seconds of etching. The sample was over-etched after 150 seconds. The microstructure of grains A and B (etching time $150 \mathrm{~s}$ ) are shown in figures 3 and 4. The ULOI curves of grains $A$ and $B$ are seen in figures 5 and 6 for different etching times (0,55 and $150 \mathrm{~s})$. The maximum observed in $80^{\circ}$ and $260^{\circ}$ corresponds to the main direction of the mechanical grinding (etching time $0 \mathrm{~s}$ ). The angular position of the peaks varies as a function of the etching time, new peaks appear in other positions, due to the modification of surface topography. This result is clearly seen in grain $\mathrm{B}$.

The TEC curves of grains A and B are indicated in figure 7. Both curves are similar. The first maximum is seen at $6 \mathrm{~s}$ and the second one between 57 and 59 s. Two minima appear in 16-19 s and 103-106 s.

\section{Aluminium}

We analyzed the damage done by the Keller reagent (figure 8) and the caustic reagent (figure 9) on the surface of grain C. ULOI curves were obtained for different etching times between 0 and 200 s. Figure 10 shows the TEC curves, for the first 200s of chemical etching, for both solutions. Clearly, both TEC curves are different from those obtained with zinc. A constant value predominates after $40 \mathrm{~s}$ of Keller etching. In the case of the caustic solution, the TEC curve has a maximum value around 20-25 s, and then it drops.

\section{Discussion}

In the case of zinc, for $\mathrm{t}=0 \mathrm{~s}$, the TEC value is different because both grains have different crystalline orientations. For $t>0 s$, the TECs have similar tendency, similar $t_{M}$ (approximately $6 \mathrm{~s}$ and 58 s) due to the material and not to the orientation.

In the case of aluminium, for $t=0 \mathrm{~s}$, the TEC value is almost the same due to the fact that we are studying the same grain with 


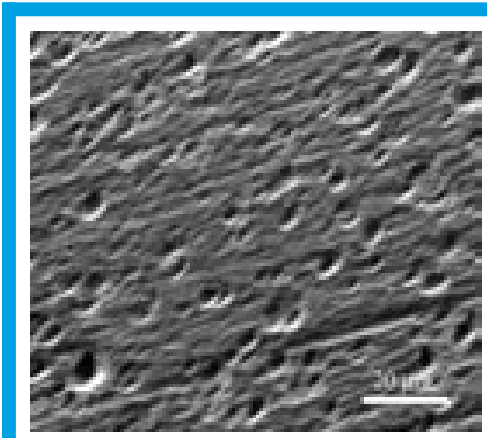

Figure 8: Aluminium sample after chemical etching with Keller reagent. Crystalline grain C. SEM

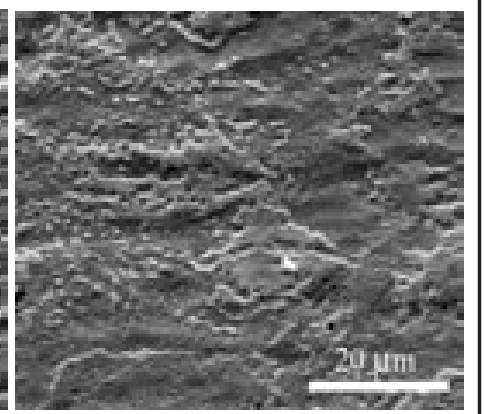

Figure 9: Aluminium sample after chemical etching with caustic reagent. Crystalline grain C. SEM

the same mechanical preparation. For $t>0$ s, the TEC curves have different morphology, due to the fact that we are using two different solutions with different components.

It is known from theory that scattering is strongly dependent on particle size, so that maximum scattering occurs at a particle size of the same magnitude as the radiation wavelength. Our TEC curves show maxima, which means high laser intensity scattered by the surface and gathered by the objective of the microscope $\left(85 \sim 90^{\circ}\right.$ from the incident beam), at different etching times. As a first approach, it may be argued that at those times, certain topographic features

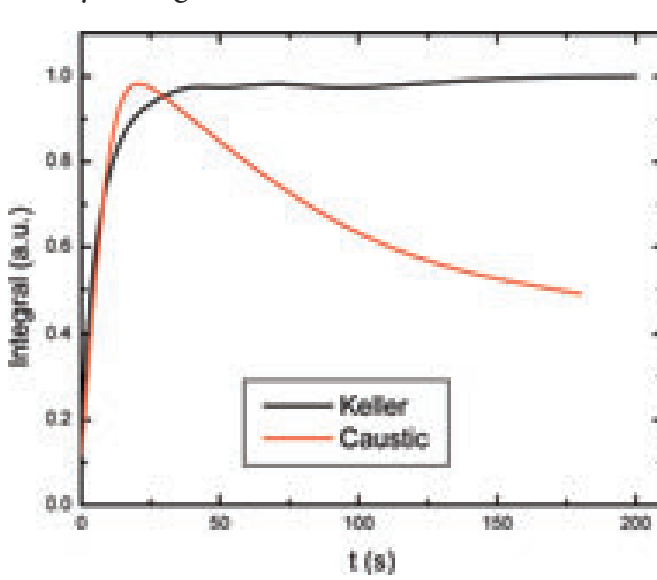

Figure 10: TEC curves of grain C. of the surface - with dimensions - similar to the la- ser wavelength - $(632.8 \mathrm{~nm})$ com- monly appear, - due to chemical - etching. Another simplified expla- nation could be to consider the topography as a superposition of diffraction gratings in different angular directions (Favret and Povolo, 2001) and at those times when the TEC curves show maxima, the number of gratings appearing in different angular directions is maximum. For example, in figure 8 the set of etch pits could be seen as diffraction gratings in two or three angular directions (sides of the etch pit), in figure 9 this kind of structure is not seen. The former has a TEC curve with higher values than the latter.

Even though more studies need to be done to find the correct explanation of the observed effects, these first results show a new way to analyze how the micro-topography of a surface evolves as a function of the damage produced by the chemical etching.

\section{References}

Favret, E., Povolo, F. and Canzian, A. (1999): Determination of Crystal Orientations in Aluminium by Means of Unidirectional Laser Oblique Illumination (ULOI). Practical Metallography. $\underline{36}, 206-215$.

Favret, E. and Povolo, F. (2001): The linear rugosity concept of crystalline surfaces by using the Unidirectional Laser Oblique Illumination (ULOI) technique, Microscopy Research and Technique. 55, No. 4, 270-281.

Favret, E. (2002): Analysis of the Intensity Curves obtained by the Unidirectional Laser Oblique Illumination (ULOI) at different magnifications, Microscopy and Microanalysis. $\underline{8}, 182-190$.

\section{Find New \& Used Microscopes}

\section{Join over 120,000 members}

at

- Search over 1,250 microscope ads currently online

๑ Buy $\&$ sell all major brands

- Free "wanted" ads for quick results

- Sell with no commission or final value fees

\section{Over 325 equipment categories including:}

Microscopes

Microscopes Accessories

Clinical Microscopes

Electron Microscopes

Image Analysis

Microtomes

Histology/Pathology

Semiconductor

And Also...

Balances

Glassware

HPLC

GC

Mass Specs

Pipettors

Mills/Grinders

Spectrophotometers

And More!

mICROSCOPY TODAY July 2007 